\title{
Signal Transmission in Chemical Systems: Propagation of Chemical Waves through Capillary Tubes
}

\author{
Ägota Tóth, Vilmos Gáspár, ${ }^{\dagger}$ and Kenneth Showalter \\ Department of Chemistry, West Virginia University, Morgantown, West Virginia 26506-6045
}

Received: August 16, 1993; In Final Form: October 19, $1993^{\circ}$

\begin{abstract}
The propagation of chemical waves through narrow channels has been investigated. Thin layers of excitable Belousov-Zhabotinsky mixtures are connected by precision-bore capillary tubes of different internal diameters. A wave initiated on one side of an otherwise impenetrable barrier enters and travels through the capillary tube, forming a hemisphere of excited solution at the exit. When the tube diameter is greater than a critical value, the excitation serves to initiate a circular wave in the second compartment; otherwise, the hemisphere collapses and no wave is initiated. Electrochemically generated periodic wave trains give rise to resonance patterns characterized by firing numbers $1 / n$, where $n=1,2$, etc. is the number of waves entering the tube for every wave exiting. These firing numbers correspond to one branch of a Farey tree; higher periodic resonances in modeling calculations indicate that more fully developed Farey sequences may also occur. A one-dimensional mapping procedure is proposed to describe the appearance and ordering of the resonance patterns.
\end{abstract}

\section{Introduction}

Signal transmission in biological systems commonly occurs via excitable media. ${ }^{1-3}$ An action potential, for example, propagates down an axon when the neuron receives a stimulus (or stimuli) above some critical threshold. The continuation of the signal requires a relay of the axon excitation across synaptic connections to other neurons, which also fire if sufficiently stimulated. Waves of activity in excitable tissue, e.g., waves of electrical excitation in the heart, also occur in response to abovethreshold stimuli. Depending on the system, an excitatory response may occur with one stimulus or may require multiple stimuli.

We use Belousov-Zhabotinsky (BZ) solutions to investigate the transmission of signals between locally coupled excitable systems. The coupling is via a precision-bore capillary tube spanning an otherwise impenetrable barrier between thin films of excitable reaction mixture. How the signal-in the form of an electrochemically initiated periodic wave train-is transmitted from one compartment to the other depends on signal frequency, tube internal diameter (i.d.), and composition of the medium. For a particular, reaction mixture, the tube i.d. must be greater than a critical value for any wave to be transmitted. This limit is a measure of the critical nucleation size for the medium and is a function of the reactant concentrations. Waves are initiated in the second compartment for tube i.d.'s above the critical value, but in a 1:1 manner only for significantly larger diameters. For tube i.d.'s near the critical value, $1 / n$ resonance patterns are exhibited, with one wave initiated in the second compartment for every $n$ waves entering the capillary tube in the first compartment.

Chemical waves appear spontaneously in thin layers of BZ reaction mixtures as concentric rings emanating from pacemaker centers. The wave source can usually be traced to the site of a dust particle or a scratch in the glass reaction vessel..$^{1,4,5}$ Waves may also be electrochemically initiated by momentarily depleting bromide at a positively biased silver electrode. ${ }^{6}$ The reaction is locally switched to the autocatalytic production of bromous acid, the outward diffusion of which gives rise to the propagating wave. It is likely that the spontaneous wave sources also rely on the local depletion of bromide, presumably by adsorption onto a

\footnotetext{
- To whom correspondence should be addressed.

† Permanent address: Department of Physical Chemistry, Kossuth L. University, P.O. Box 7, 4010 Debrecen, Hungary.

- Abstract published in Advance ACS Abstracts, December 15, 1993.
}

heterogeneous particle or imperfection in the vessel wall. A local depletion of molecular bromine, a species that readily adsorbs to surfaces, would also serve to initiate the autocatalytic generation of bromous acid.

The initiation of a chemical wave depends on switching the reaction to the excited state; however, it also depends on the size of the region in that state. Because the diffusive dispersion of bromous acid is enhanced in a convex wave front, there is a critical nucleation size below which the outward diffusion of the autocatalyst is insufficient to sustain wave advancement. The critical size can be estimated by considering the effect of curvature on the velocity of front propagation. Using singular perturbation theory, Tyson and Keener ${ }^{7-9}$ showed that the normal velocity $N$ of a circular wave is proportional to the front curvature $x$ according to the eikonal equation

$$
N=c+D k
$$

where $c$ is the planar wave velocity and $D$ is the diffusion coefficient of the autocatalyst. The velocity of waves with positive curvature (concave in the direction of propagation) is greater than the planar wave velocity, and with negative curvature (convex in the direction of propagation) the velocity is smaller. The radius of a circular region corresponding to the critical nucleation size is given by eq 1 when the normal velocity $N=0$,

$$
r_{\mathrm{c}}=-\frac{1}{\kappa}=\frac{D}{c}
$$

In typical $\mathrm{BZ}$ reaction mixtures, the critical radius estimated from the planar velocity is on the order of $50 \mu \mathrm{m} .{ }^{7}$ Nagy-Ungvarai et al. ${ }^{10}$ used fine silver wires and silver-coated capillaries to initiate waves and were able to bracket the critical nucleation radius between two values in a series of $\mathrm{BZ}$ solutions. We have carried out wave initiation experiments using silver-loaded cationexchange beads. Critical bead sizes, above which waves were initiated and below which no initiation occurred, were determined for a range of reaction mixture compositions. The critical values were in qualitative agreement with the earlier studies using silver wires. Both of these methods, however, suffer from an uncertainty associated with the thickness of the layer of silver ions produced by the wire or bead. The precision-bore capillary tubes used in this study allow the measurement of critical nucleation size without this uncertainty.

In the first part of this report we describe experiments in which capillary tubes are used to directly measure the critical nucleation 
TABLE 1: Reagent Concentrations

$\begin{array}{ll}\mathrm{H}_{2} \mathrm{SO}_{4} & 0.14 \mathrm{M} \\ \text { malonic acid } & 0.02 \mathrm{M} \\ \mathrm{NaBrO}_{3} & 0.10-0.15 \mathrm{M} \\ \text { catalyst }^{a} & 9.0 \times 10^{-4} \mathrm{M}\end{array}$

- Tris(bathophenanthrolinedisulfonato)iron(II).

size in excitable $\mathrm{BZ}$ solutions. The measured values are compared with values predicted by the eikonal equation and with the results of earlier investigations. ${ }^{10-12}$ In the second part, we report on experiments in which successive waves enter and propagate through the capillary tube. Resonance patterns are found between the number of waves entering and the number exiting the tube. The propagation of chemical waves through narrow gaps has been studied using Fitzhugh-Nagumo and Ginzburg-Landau models, ${ }^{13-16}$ and $1 / n$ resonances as well as more complex patterns were found. We use the two-variable Tyson-Fife ${ }^{5}$ model to numerically investigate $\mathbf{B Z}$ waves propagating through narrow channels. A one-dimensional mapping procedure is also introduced to describe the sequences of resonance patterns.

\section{Experimental Section}

Solutions. Belousov-Zhabotinsky reaction mixtures were prepared with the concentrations given in Table 1. All reagent solutions were made with analytical grade chemicals and subsequently filtered through $0.45-\mu \mathrm{m}$ membrane filters (Millipore). The catalyst was prepared from iron(II) sulfate and bathophenanthrolinedisulfonic acid disodium salt (Aldrich) in a 1:3 mole ratio. The color of a $\mathrm{BZ}$ mixture with this iron complex changes from dark red in the reduced state to bright green in the oxidized state.

Experimental Setup and Procedures. New reaction mixtures were prepared for each experiment. An experiment was initiated by stirring the solution until an oxidation cycle was induced. ${ }^{6} \mathrm{~A}$ $10.0-\mathrm{mL}$ aliquot of the solution was then spread uniformly into a flat polycarbonate Petri dish (10-cm diameter, Nalgene) resulting in a depth of $\sim 1.3 \mathrm{~mm}$. The dish was divided into two compartments by a rectangular block of Plexiglas through which a short length $(\sim 6.0 \mathrm{~mm})$ of capillary tube (Polymicro Technology) was mounted. The polymer coating of the flexible capillary tube was first removed by gentle heating with a Bunsen burner. The glass tube was then mounted in a hole matching the outside diameter, drilled through the Plexiglas block. Acetone solvent was used to fuse the Plexiglas around the glass tube. A gap in the Plexiglas block near the wall of the Petri dish allowed the liquid levels in each compartment to quickly equalize. A Plexiglas top covering the dish prevented disturbances of the solution by air currents and also served as a holder for the wavegeneration electrodes. Experiments were carried out with the Petri dish in a thermostated stage maintained at $25.0 \pm 0.1^{\circ} \mathrm{C}$. Each reaction mixture was examined for a maximum of $2 \mathrm{~h}$ and then discarded.

Wave Generation. Waves were electrochemically initiated after a 6.0-min period following the induced oxidation cycle. A silver wire electrode (1.00-mm diameter, Aldrich), negatively biased at $-1.2 \mathrm{~V}$ with respect to a platinum wire electrode, was positively biased at $+2.0 \mathrm{~V}$ for $100 \mathrm{~ms}$ to initiate a wave. The pulses were triggered at preset intervals by an arbitrary waveform generator (Keithley/Metrabyte PCIP-AWFG).

Image Processing. The solution was illuminated from below by diffuse light passing through a broad band interference filter (500-nm peak transmittance, Oriel). The video camera (MTI) was attached to a zoom microscope (Zeiss), allowing the region of solution around the capillary tube to be imaged at different magnifications. The images were recorded on video tape or stored digitally on hard disk using a frame grabber (Matrox, MVPAT). Successive digital images at 5-s intervals, enhanced by standard techniques such as image subtraction, allowed highresolution tracking of the wave front position.

\section{Experimental Results}

Critical Radius. A capillary tube provides a narrow channel for the propagation of chemical waves between otherwise separated $\mathrm{BZ}$ solutions. When a wave is initiated at one side of the barrier, it enters the tube and travels along the conduit of excitable reaction mixture. Figure 1 shows subtraction images at 5-s intervals of a wave traveling from left to right inside a capillary tube of 160 $\mu \mathrm{m}$ i.d. On exiting the tube, the wave forms a hemisphere of excited solution at the opening. Whether this hemisphere serves as a wave source or simply decays depends on the curvature of the wave front.

The eikonal equation for a wave front in three spatial dimensions is obtained by generalizing eq 1 :

$$
N=c+D\left(\kappa_{1}+\kappa_{2}\right)
$$

where $\kappa_{1}$ and $\kappa_{2}$ are the principal curvatures. ${ }^{7}$ Assuming a perfect hemisphere $\left(\kappa_{1}=\kappa_{2}=\kappa\right)$, the critical radius is given by

$$
r_{\mathrm{c}}=-\frac{1}{\mathrm{~K}}=\frac{2 D}{c}
$$

According to eqs 3 and 4 , a hemisphere of excitation becomes a nucleation center if its radius is greater than the critical value. The last frame in Figure 1 shows that the hemisphere is large enough to initiate a propagating wave in the second compartment. As the region of excitation expands and the system recovers from its excited state, a circular wave travels outward from its epicenter at the capillary tube opening. Because the radius of the initial hemisphere is effectively that of the capillary tube, the critical nucleation radius for this particular solution composition must be less than the $80-\mu \mathrm{m}$ inner radius of the tube. Experiments with smaller tubes show that for these reactant concentrations, $37.5 \mu \mathrm{m}<r_{\mathrm{c}}<50 \mu \mathrm{m}$.

Experiments with the commercially available tube sizes did not allow an accurate determination of the critical radius; therefore, a different approach was taken. The critical radius is inversely proportional to $c$, the planar wave velocity, which is a function of the composition of the system. By systematically changing the solution composition until waves are no longer transmitted from one compartment to the other, the critical radius corresponding to a particular composition can be determined. The dependence of wave velocity on bromate and sulfuric acid concentrations in various $\mathbf{B Z}$ reaction mixtures has been found to have the form ${ }^{17-21}$

$$
c=A+B\left(\left[\mathrm{H}_{2} \mathrm{SO}_{4}\right]\left[\mathrm{BrO}_{3}^{-}\right]\right)^{1 / 2}
$$

The coefficients $A=-2.13 \mathrm{~mm} \mathrm{~min}^{-1}$ and $B=28.2 \mathrm{~mm} \mathrm{~min}^{-1}$ $\mathrm{M}^{-1}$ were determined from measurements of wave position as a function of time in a series of six reaction mixtures, with bromate concentration varying over the range in Table 1 . The values are in reasonably good agreement with the measurements of previous studies. ${ }^{17-21}$

In a series of experiments with varying $\left[\mathrm{BrO}_{3}^{-}\right]$, a critical concentration between 0.1050 and $0.1075 \mathrm{M}$ was determined for the transmission of waves through a tube of $160-\mu \mathrm{m}$ i.d. Figure 2 shows subtraction images at 5-s intervals of a wave traveling inside the tube for $\left[\mathrm{BrO}_{3}^{-}\right]=0.100 \mathrm{M}$. At this concentration, slightly lower than the critical value, the wave readily enters and propagates within the tube; on reaching the tube opening, however, the hemisphere of excitation collapses, as shown in the last frame. The transmission of wave activity from one compartment to the other is now inhibited.

A comparison of the critical radius measurements with values from other studies can be made by calculating the diffusion coefficient in eq 4 using the measured values of $c$ and $r_{\mathrm{c}}$. The 


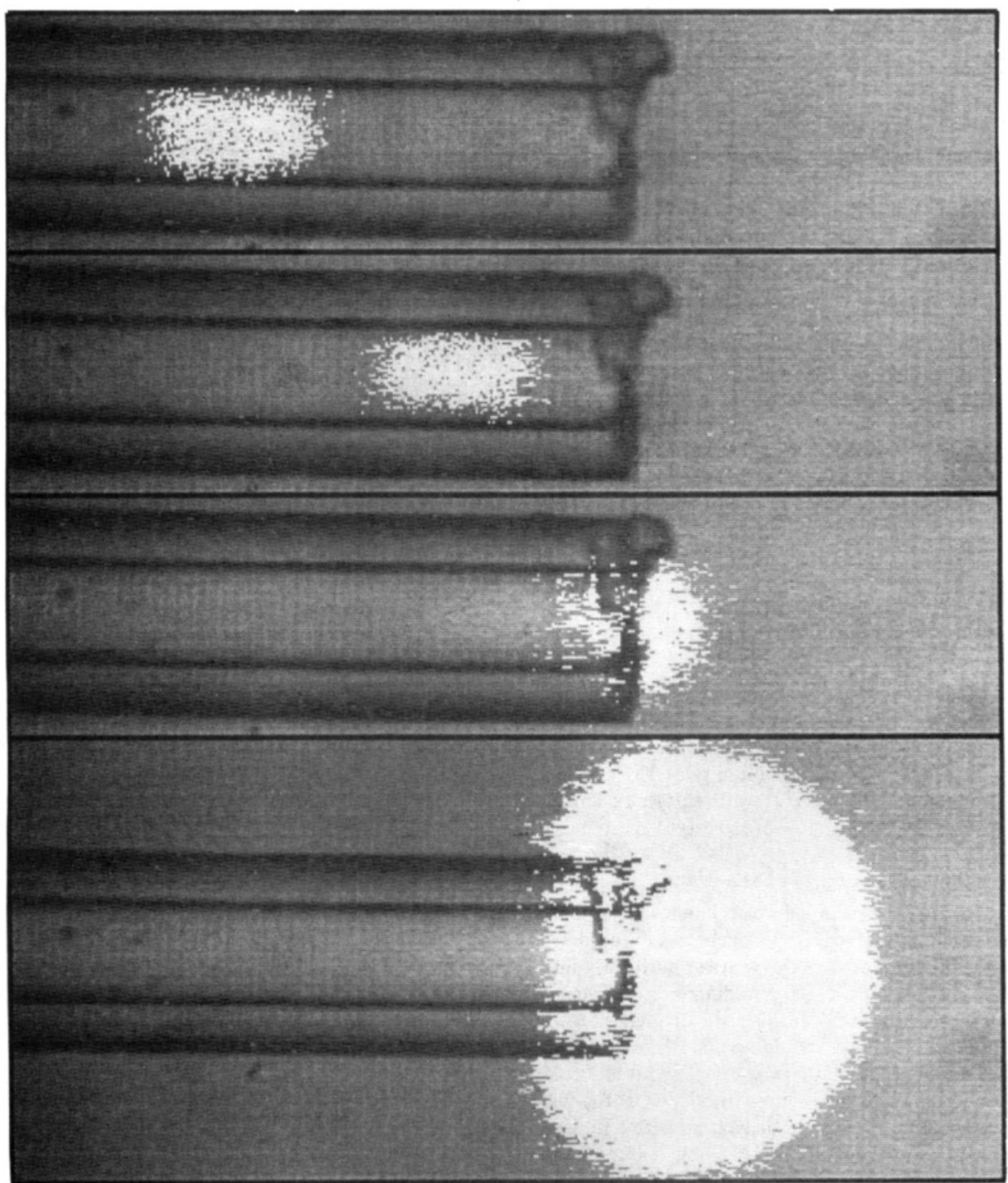

Figure 1. Subtraction images showing a chemical wave at 5 -s intervals traveling inside a capillary tube of $160-\mu \mathrm{m}$ i.d. The wave grows into a hemisphere at the tube opening, which then generates an expanding circular wave. Concentrations are given in $\mathrm{Table} 1$ with $\left[\mathrm{BrO}_{3}^{-}\right]=0.15 \mathrm{M}$; field of view in the bottom panel is $1.45 \mathrm{~mm} \times 0.71 \mathrm{~mm}$.

planar velocity calculated from eq 5 with $\left[\mathrm{BrO}_{3}^{-}\right]=0.1075 \mathrm{M}$ gives $c=2.216 \times 10^{-2} \mathrm{~mm} \mathrm{~s}^{-1}$. With the corresponding critical radius of $80 \mu \mathrm{m}$, eq 4 gives a value of $8.86 \times 10^{-6} \mathrm{~cm}^{2} \mathrm{~s}^{-1}$ for the diffusion coefficient. Foerster et al. ${ }^{11,12}$ have also used velocity measurements with the eikonal relation to deduce the value of $D$. They found values of $1.996 \times 10^{-5} \mathrm{~cm}^{2} \mathrm{~s}^{-1}$ and $1.903 \times 10^{-5}$ $\mathrm{cm}^{2} \mathrm{~s}^{-1}$, respectively, from the cusp formed by two colliding circular waves and from very small expanding circular waves. The value of $D$ from our measurements is significantly lower than these values (and an expected value between $1 \times 10^{-5}$ and $2 \times 10^{-5} \mathrm{~cm}^{2}$ $\mathrm{s}^{-1}$ typical for small molecules). Field and Noyes ${ }^{17}$ estimated a value of $1.8 \times 10^{-5} \mathrm{~cm}^{2} \mathrm{~s}^{-1}$ to model their wave velocity measurements, and a value of $1.5 \times 10^{-5} \mathrm{~cm}^{2} \mathrm{~s}^{-1}$ was used by Ross and co-workers 22 to model their concentration profile measurements. Kuhnert et al. ${ }^{23}$ argued that the value of $D$ should be around $1.3 \times 10^{-5} \mathrm{~cm}^{2} \mathrm{~s}^{-1}$, close to the measured values for $\mathrm{LiClO}_{4}$, $\mathrm{NaClO}_{4}$, and $\mathrm{KIO}_{3}$, because of the similarity of $\mathrm{HBrO}_{2}$ to these species.

There are several possible reasons for the discrepancy between the value of $D$ from our measurements and the values from other studies. It is first important to recognize that there is no reason to expect the eikonal equation to quantitatively predict the critical nucleation size, since the theoretical development of the equation requires the assumption of slight curvature. ${ }^{7-9}$ On the other hand, the eikonal equation has been remarkably successful in describing curvature effects in propagating waves, and moreover, it provides a basis for comparisons between different curvature measurements. We therefore searched for possible experimental errors that could account for our low value of $D$ (due to a low value for the measured critical radius). Figures 1 and 2 show that the ends of the glass tubes are quite irregular, which certainly makes the assumption that the tube radius is effectively the radius of the hemisphere of excited solution only an approximation. A more important possibility is that the chemical environment of the solution inside the tube (and at the tube opening) is different from the bulk solution. This suspicion was confirmed when velocities of waves inside the capillary tubes were measured. Measurements from videotapes and digitally stored images, as well as new experimental measurements, showed that wave velocities inside the tubes are significantly higher than those in the corresponding bulk solution. Wave velocities inside the tubes shown in Figures 1 and 2 were respectively $51 \%$ and $42 \%$ higher 


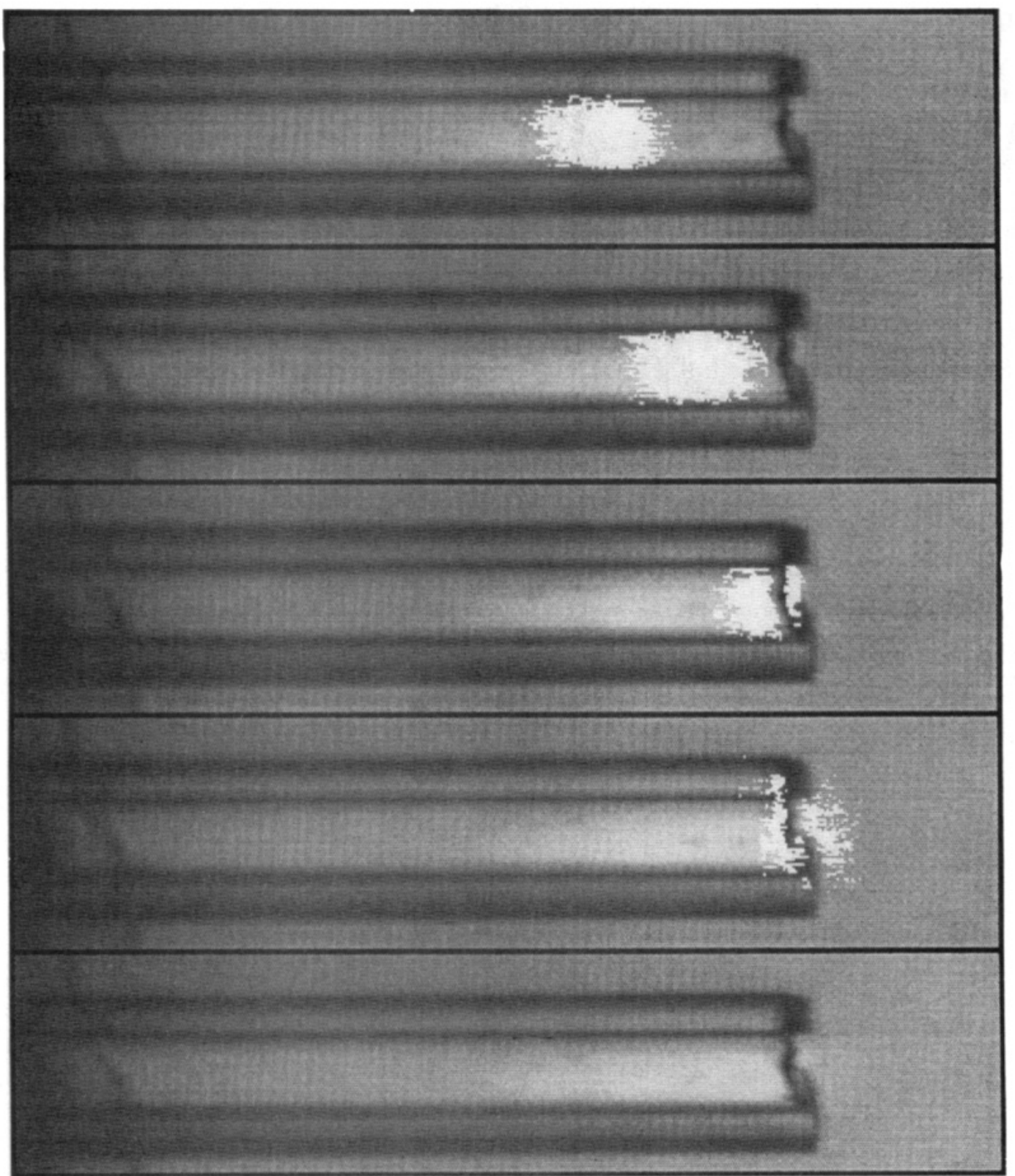

Figure 2. Subtraction images showing a chemical wave at 5 -s intervals traveling inside a capillary tube. Conditions and the tube inside diameter are the same as in Figure 1 except $\left[\mathrm{BrO}_{3}^{-}\right]=0.10 \mathrm{M}$. At this bromate concentration, the tube size is below the critical size for wave nucleation, the hemisphere of excitation collapses, and no traveling wave develops. The field of view in each panel is $1.9 \mathrm{~mm} \times 0.44 \mathrm{~mm}$.

than the bulk solution wave velocities. New measurements at the critical $\mathrm{BrO}_{3}$ - concentration of $0.1075 \mathrm{M}$ showed an average wave velocity within the tube $43 \%$ higher than the velocity predicted by eq 5 . If we assume that the velocity within the tube determines the critical nucleation size, a diffusion coefficient of $1.27 \times 10^{-5} \mathrm{~cm}^{2} \mathrm{~s}^{-1}$ is calculated from eq 4 . It is not clear how the chemical environment is altered by the glass tube to cause the increase in wave velocity. A plausible explanation is that the high surface to volume ratio affects the reaction mixture, perhaps by adsorption of $\mathrm{Br}^{-}$or $\mathrm{Br}_{2}$, thereby affecting the velocity.

Resonance Patterns. The systematic variation of bromate concentration allows the examination of wave behavior very close to the critical nucleation size. According to eq 3, the normal wave velocity $N$ approaches zero as the (negative) curvature approaches a critical value. This has important consequences for the transmission of periodic wave trains from one side of the barrier to the other when the tube radius is near $r_{\mathrm{c}}$. The decrease in propagation velocity at the tube exit due to the wave curvature influences the propagation of subsequent waves. An example of the effect is shown in Figure 3, where the periodic wave train initiated in the left compartment gives rise to a wave train in the right compartment with a period twice as long. This pattern arises because only every other wave of the original wave train is transmitted to the second compartment. The behavior can be viewed as the spatiotemporal analogue of periodic resonances in forced excitable systems in well-stirred, open reactors. ${ }^{24-27}$ We exploit this resemblance and characterize the patterns in terms of the firing number fn, defined by the number of exiting waves divided by the number of entering waves. For this solution composition and tube i.d., $\mathrm{fn}=1 / 2$, with the wavelength beyond the barrier twice that of the electrochemically initiated wave train.

Measurements of gray level (proportional to transmitted light intensity) at points near each end of the capillary tube are shown in Figure 4. The time trace for the entering waves, with period $T_{\mathrm{f}}=200 \mathrm{~s}$ set by the electrochemical wave generator, is shown in Figure 4a; the trace for the exiting waves is shown in Figure $4 \mathrm{~b}$, in which the period is $2 \times T_{\mathrm{f}}$. The solution composition and tube i.d. are the same as in Figure 3. Shown in Figure 5 are time traces for entering and exiting waves with an initiation period $T_{f}$ $=150 \mathrm{~s}$. The tube i.d. is the same as in Figure 4; however, now $\mathrm{fn}=1 / 3$ due to the higher frequency of waves entering the tube 


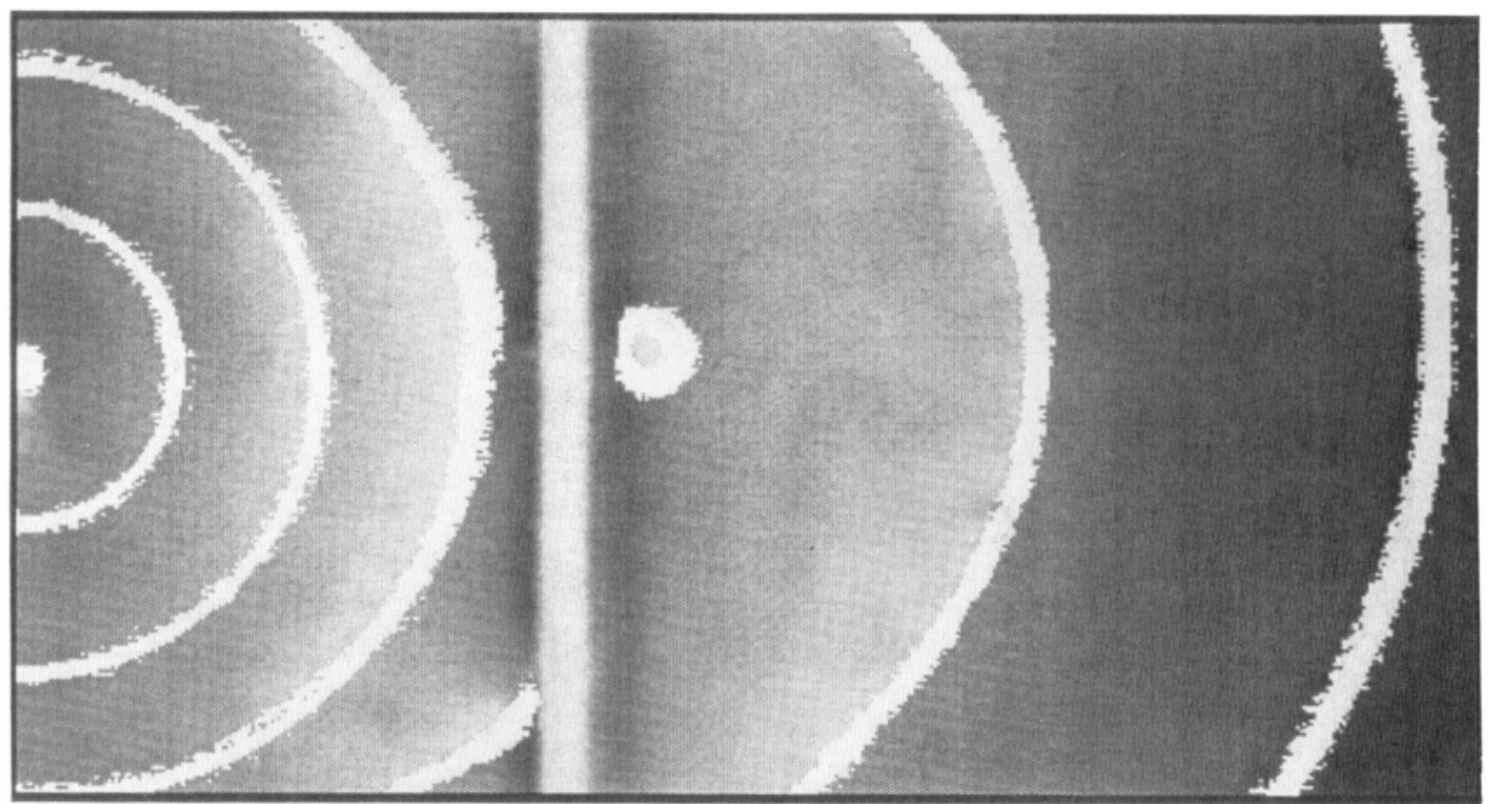

Figure 3. Subtraction images showing thin films of solution in two compartments separated by a Plexiglas barrier. Waves initiated electrochemically in the left compartment enter the capillary tube positioned at the center of the barrier. Each entering wave propagates through the tube; however, only every other wave results in wave initiation in the right compartment. The result is a 1:2 resonance pattern. The field of view is $5.0 \mathrm{~cm} \times 2.7$ $\mathrm{cm}$; concentrations are given in Table 1 with $\left[\mathrm{BrO}_{3}{ }^{-}\right]=0.14 \mathrm{M}$.


Figure 4. Time series for 1:2 resonance pattern. Gray level values (on a scale of 256 units) from images of waves entering (a) and exiting (b) a tube of $160-\mu \mathrm{m}$ i.d. The values are proportional to transmitted light intensity (at $500 \mathrm{~nm}$ ). Measurements were made at $0.2 \mathrm{~mm}$ from each end of the tube. The period of the entering waves is $T_{\mathrm{f}}=200 \mathrm{~s}$; the solution composition is the same as in Figure 3.

and a slightly lower bromate concentration. The wave speed and wavelength in both compartments show a slight drift due to the aging of the system, which is apparent in the wave spacing in Figure 3 and the maximum gray levels in Figures 4 and 5 .

A bifurcation diagram giving the dependence of firing number on entering wave period can be constructed by systematically varying the period of electrochemical wave initiation. Figure 6 shows such a bifurcation diagram for the solution composition and tube size used for Figure 5. For waves initiated at intervals greater than $375 \mathrm{~s}$, each wave exits the tube into a medium that has relaxed sufficiently from the previous wave to support wave initiation and propagation. On decreasing the period of the entering waves, a bifurcation occurs from the $1: 1$ pattern to a $1: 2$ pattern $(f n=1 / 2)$, where only every other wave is transmitted
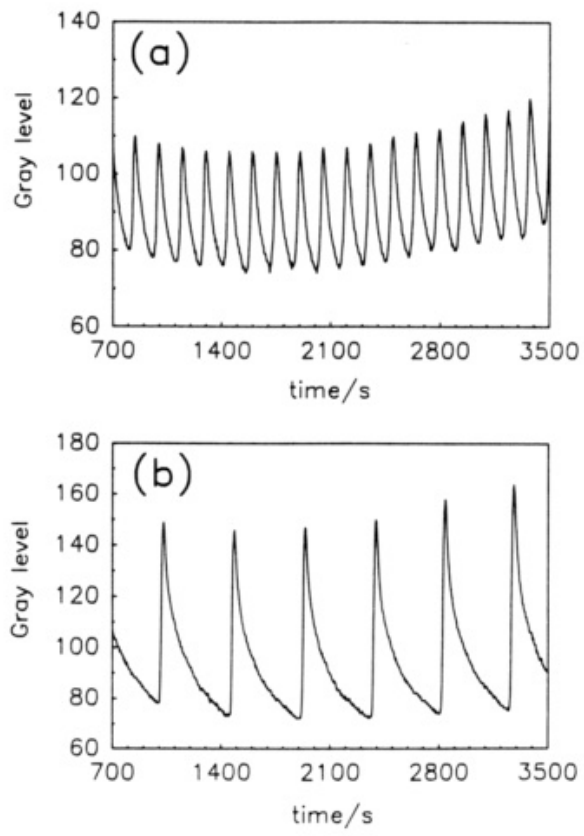

Figure 5. Time series for 1:3 resonance pattern. Measurements and experimental conditions are the same as in Figure 4 except the period of the entering waves is $T_{\mathrm{f}}=150 \mathrm{~s}$. Concentrations given in Table 1 with $\left[\mathrm{BrO}_{3}^{-}\right]=0.13 \mathrm{M}$.

to the second compartment. Every wave of the electrochemically initiated wave train enters and propagates through the tube; however, every other hemisphere of excitation at the tube opening is ineffective in producing a wave. When monitored as a sequence of subtraction images, these ineffective waves appear much like the wave shown in Figure 2. On further decreasing the entering wave period, a new pattern with the firing number $\mathrm{fn}=1 / 3$ emerges at $T_{\mathrm{f}}=175 \mathrm{~s}$. For this reaction mixture composition, the electrochemical wave initiation is limited to values above $135 \mathrm{~s}$, and no other patterns were found within this range. One might anticipate higher resonances, for example, patterns with $\mathrm{fn}=2 / 3$ or $2 / 5$ between the simple resonances $1 / 2$ and $1 / 1$ or $1 / 3$ and $1 / 2$, respectively; however, such patterns were not detected within our experimental resolution of 5-s increments in period.

The dependence of firing number on $T_{\mathrm{f}}$ was determined for a 


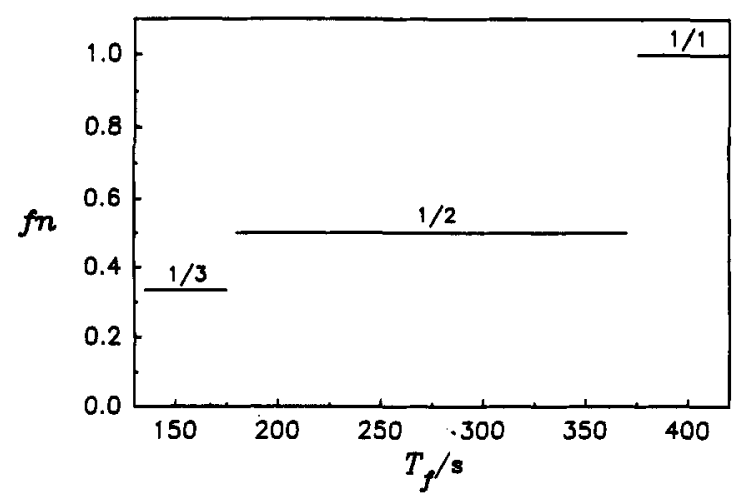

Figure 6. Bifurcation diagram showing firing number $f n$ as a function of the entering wave period $T_{\mathrm{r}}$. Concentrations are listed in Table 1 , except $\left[\mathrm{BrO}_{3}{ }^{-}\right]=0.13 \mathrm{M}$. Tube size: $160-\mu \mathrm{m}$ i.d.

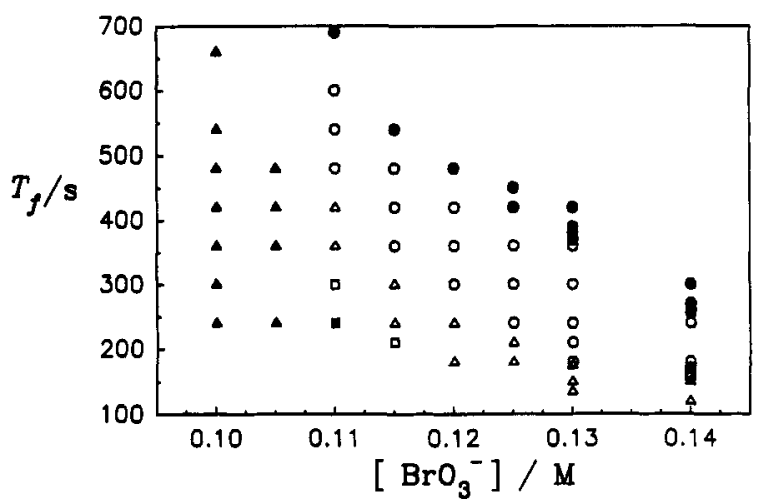

Figure 7. Phase diagram showing regions of resonance patterns for different values of period $T_{\mathrm{f}}$ and $\left[\mathrm{BrO}_{3}^{-}\right]$. Patterns are indicated by (waves exiting:waves entering) 0:1 ( $\Delta), 1: 1(\bullet), 1: 2(0), 1: 3(\Delta), 1: 4(\square)$, and $1: 5(\square)$. Tube size: $160-\mu \mathrm{m}$ i.d.

series of bromate concentrations, allowing construction of the phase diagram shown in Figure 7. As $T_{\mathrm{f}}$ is lowered, the $1: 1$ behavior gives way to $1: 2$ behavior, which occurs at lower $T_{\mathrm{f}}$ values as $\left[\mathrm{BrO}_{3}^{-}\right]$is increased. A transition from $1: 2$ to $1: 3$ behavior then occurs, and at lower $\left[\mathrm{BrO}_{3}^{-}\right]$values, $1: 4$ and $1: 5$ patterns are also exhibited. The diagram also shows the critical bromate concentration below which wave transmission from one compartment to the other does not occur.

Although most experiments were carried out with a single capillary tube and different $\mathrm{BrO}_{3}$ - concentrations, different tube sizes were used in a number of experiments. With larger tubes of 200- or 320- $\mu \mathrm{m}$ i.d., the bifurcation points for the appearance of the $1: 2$ and $1: 3$ patterns were always found at lower values of $T_{\mathfrak{f}}$. As tube size is increased, the 1:3 pattern is not observed, and with very large tubes (e.g., $540-\mu \mathrm{m}$ i.d.), the $1: 2$ pattern is not seen. Occasionally, irregular 2:3 resonances were found with the large tubes when the value of $T_{f}$ was close to the lower limit for wave initiation.

\section{Modeling Results}

Simple Resonances. In this section, we describe the simple resonances found in the capillary tube experiments in terms of a one-dimensional map. We follow earlier treatments based on circle maps of forced periodic and excitable systems. ${ }^{1,3,25,28,29}$

We first follow Glass et al. ${ }^{29}$ and consider an oscillatory system described by a set of ordinary differential equations of the form $\mathrm{d} \mathbf{x} / \mathrm{d} t=\boldsymbol{F}(\boldsymbol{x})$, where $\mathrm{x}$ is a vector of dynamic variables defining the state of the system at time $t$ and $F$ contains the nonlinear functions of the kinetics. Oscillations of period $T_{0}$ are characterized by a stable limit cycle. The phase of the oscillation $\varphi$ can be defined as $t / T_{0}$, where $t$ is the time required for the system to move to some point $x(t)$ on the cycle from some reference point $x(0)$; hence, $0 \leq \varphi \leq 1$. When a perturbation is applied to the system at some phase $\varphi$, the system is shifted away from the limit cycle to a point in phase space. The system then relaxes back to the limit cycle, and the net effect is a shift in the phase of oscillation. Such shifts can be characterized by plotting the new phase $\varphi^{\prime}$ as a function of the old phase $\varphi$ to give $g$, the phasetransition curve (PTC). This plot is constructed from experimental measurements of the period $T^{\prime}$ of the perturbed oscillation following a stimulus to the system at phase $\varphi$. If we assume that the system relaxes rapidly back to the limit cycle following the perturbation, the PTC is given by $g(\varphi)=1+\varphi-T^{\prime} / T_{0}$. If the system is perturbed periodically at an interval $T_{\mathrm{f}}<T_{0}$ and if $\varphi_{n}$ is the phase of the oscillator at the $n$th stimulus, then the phase of the system at the subsequent perturbation is given by ${ }^{29}$

$$
\varphi_{n+1}=\Psi\left(\varphi_{n}\right)=g\left(\varphi_{n}\right)+\tau
$$

where $\tau=T_{\mathrm{f}} / T_{0}$ is the dimensionless forcing period. Equation 6 defines the circle map $\Psi$, which can be iterated to compute recurring patterns with firing number $\mathrm{fn}=\mathrm{m} / \mathrm{n}$, where $m$ is the number of oscillatory cycles observed and $n$ is the number of stimuli applied.

To characterize the dynamics of periodically forced excitable systems, Marek and co-workers ${ }^{24-27}$ have developed one-dimensional mappings based on phase-excitation curves (PEC's). The PEC is analogous to the PTC, but it is constructed with the basic refractory period $T_{\mathrm{B}}$ of the excitable system rather than the period $T_{0}$ of the oscillatory system. The refractory period is the time interval required for the system to return to a small $\eta$ neighborhood of the steady state following a perturbation. The value of $T_{B}$ can be determined experimentally by applying a second perturbation at different times as the system moves around the excitation cycle. The cycle is considered to be complete and the system within the $\eta$ neighborhood of the steady state if the second perturbation causes an excitation. On the basis of this definition of excitation cycle, a map for a periodically forced excitable system is given by $^{25}$

$$
\varphi_{n+1}=\Psi\left(\varphi_{n}\right)= \begin{cases}g\left(\varphi_{n}\right)+\tau & \text { for } 0 \leq \varphi<1 \\ \tau & \text { for } \varphi \geq 1\end{cases}
$$

where now the dimensionless forcing period $\tau$ is defined as $T_{\mathrm{f}} / T_{\mathrm{B}}$ and the function $g\left(\varphi_{n}\right)$ is the PEC.

Marek et al. ${ }^{25}$ showed that such an interrupted circle map describes $1 / n$-type resonances when $g\left(\varphi_{n}\right)$ is a monotonously increasing function of $\varphi$. The discontinuity of the map follows from the assumption that once the $\eta$ neighborhood of the steady state is reached the phase is reset to zero. The system then remains at rest until the next stimulus results in an above-threshold perturbation, thus initiating a new cycle.

The effect of a periodic wave train passing through a capillary tube is much like the periodic forcing of an excitable system, only now the perturbation occurs locally from the transmitted chemical wave. Resonance patterns occur when waves propagating through the capillary tube cannot excite the solution at the exit because it is in the refractory period of its excitation cycle. We use a one-dimensional map in the form of eq 7 to describe the simple resonance patterns observed in our experiments. However, $T_{f}$ now refers to the period of the waves entering the tube, while $g\left(\varphi_{n}\right)$ represents the PEC of an excitable solution element at the exit of the tube.

An iteration of the map such that $\varphi_{n+1} \geq 1$ corresponds to the system (at the tube exit) relaxing to the $\eta$ neighborhood of the steady state. The value of $g\left(\varphi_{n+1}\right)$ is set to zero in this case because the phase is reset by the relaxation. The following iteration always represents an above-threshold perturbation, where a wave is initiated at the exit of the tube. Since the second expression in eq 7 adds a constant in the mapping, successive iterations always lead to periodic solutions. As the value of $T_{f}$ is varied, different periodic solutions appear, depending on the position of the map with respect to the bisectrix (given by $\varphi_{n+1}=\varphi_{n}$ ). At large values 


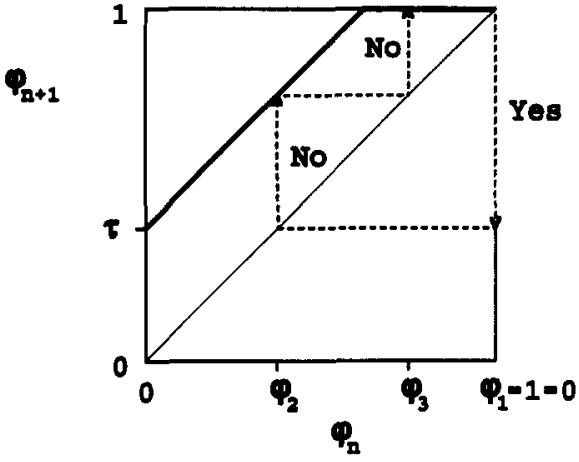

Figure 8. Successive iterations of map based on eq 7 for $1: 3$ resonance pattern.

of $T_{f}$, such that $\tau \geq 1$, every entering wave generates an exiting wave. The solution at the exit of the tube has enough time to recover before the next entering wave arrives. On decreasing the period of the entering waves, the value of $\tau$ decreases and the map is shifted toward the bisectrix. For $\tau<1$, more than one wave can enter the tube for each wave initiated at the exit and different $1 / n$ resonance patterns develop.

The mapping of a 1:3 pattern is illustrated in Figure 8. The form of the PEC in the solution element at the exit of the tube is not known; however, the experimental measurements (discussed below) indicate that the amplitude of the perturbation due to an incoming wave is sufficiently small that the phase shift on the excitation cycle is negligible $\left(\varphi^{\prime}=g(\varphi)=\varphi\right)$. We therefore assume that the map $\Psi$ is simply a straight line parallel to the bisectrix; i.e., only the timing of the entering waves is important. The intercept of the map (at $\varphi_{n}=0$ ) is the dimensionless period $\tau$ of the entering waves. The evolution of the 1:3 resonance pattern is described by the following mapping sequence:

$$
\begin{aligned}
\varphi_{1}=0 \longrightarrow \underset{\text { Yes }}{\longrightarrow} \varphi_{2}=\tau \longrightarrow \underset{\text { No }}{\longrightarrow} \varphi_{3}=2 \tau \underset{\text { No }}{\longrightarrow} \\
\varphi_{4}=1 \Longrightarrow \varphi_{1}=0 \underset{\text { Yes }}{\longrightarrow} \varphi_{2}=\tau \underset{\text { No }}{\longrightarrow} \text { etc... }
\end{aligned}
$$

where $\varphi_{1}, \ldots, \varphi_{4}$ are the phases of the solution element at the exit of the tube when the incoming waves arrive. Yes and No indicate whether or not a wave is initiated at the exit. The symbol $\Rightarrow$ represents a phase-resetting event, i.e., the effectively complete relaxation of the system at the tube exit.

The closed loop of dashed lines in Figure 8 illustrates the periodic solution of the subsequent iterations of the map. The number of vertical arrows gives the number of stimuli $(n=3)$ due to the entering waves. Arrows pointing upward correspond to ineffective exiting waves and the arrow pointing downward corresponds to a wave initiation. Thus, the stable periodic solution is a $1: 3$ resonance pattern.

With a linear PEC parallel to the bisectrix, the mapping procedure gives $1: 2$ patterns in the range $1 / 2 \leq \tau<1$ and $1: 3$ patterns in the range $1 / 3 \leq \tau<1 / 2$. Evidence that a negligible phase shift occurs when a wave exits the capillary tube can be seen in Figure 6. The largest value of $T_{f}$ for the 1:2 pattern corresponds to the basic refractory period $T_{B}$ of the excitable system. If there were no phase shift at all, we would expect the largest value of $T_{\mathrm{f}}$ for the 1:3 pattern to be $1 /{ }_{2}\left(T_{\mathrm{B}}\right)$, which from Figure 6 is $1 / 2(370 \mathrm{~s})=185 \mathrm{~s}$. This compares with $T_{\mathrm{f}}=175 \mathrm{~s}$ for the largest period of entering waves giving the $1: 3$ pattern. The most important feature in determining the resonance patterns is the timing of the entering waves with respect to the excitation cycle. The small phase shift has little effect, and to a good approximation the PEC is linear.

Other resonance patterns appear, e.g., 1:4, 1:5, etc. as the incoming wave period $T_{\mathrm{f}}$ is decreased and the PEC moves closer to the bisectrix. The range of $T_{f}$ is the limiting factor in determining how many of the patterns are observed. The lower limit of $T_{f}$ is dependent on the rate at which waves can be electrochemically initiated, which is dependent on the solution composition. No resonances beyond the 1:3 pattern could be observed in Figure 6 due to this limit; however, as shown in Figure $7,1: 4$ and $1: 5$ patterns were found at lower $\mathrm{BrO}_{3}{ }^{-}$concentrations.

The basic refractory period $T_{\mathrm{B}}$, experimentally determined as the upper limit of the $1: 2$ pattern, increases with decrease in tube size. This trend is consistent with the effects of curvature on the exiting wave. As tube size decreases and curvature increases, the velocity of the exiting wave decreases due to enhanced dispersion of autocatalyst in the wave front. The effective period of the excitation cycle therefore increases at the exit of the tube. In the limit of $r=r_{c}$, the wave velocity becomes zero and the hemisphere of excitation collapses. In terms of eq 7, as $r \rightarrow r_{\mathrm{C}}, T_{\mathrm{B}} \rightarrow \infty$ and $\tau \rightarrow 0$; therefore, the transmission of waves at the exit of the tube cannot occur, as $\varphi_{n+1}=\varphi_{n}=0$. In the limit of $r \rightarrow \infty$, where the wave becomes effectively planar, every entering wave can initiate a wave since $\tau \geq 1$. Between these extremes, the value of $T_{\mathrm{B}}$ increases as the tube size decreases; the map is shifted toward the bisectrix, and different resonance patterns develop.

Numerical Modeling. Wave transmission through narrow channels was also investigated numerically using the Tyson and Fife $^{5}$ reduction of the Oregonator model ${ }^{30}$ for the $\mathrm{BZ}$ reaction. The spatiotemporal behavior is described by the partial differential equations

$$
\begin{aligned}
& \frac{\partial u}{\partial t}=\nabla^{2} u+\frac{1}{\epsilon}\left[u(1-u)-f v \frac{u-q}{u+q}\right] \\
& \frac{\partial v}{\partial t}=\nabla^{2} v+(u-v)
\end{aligned}
$$

where the fast variable $u$ and the slow variable $v$ represent the dimensionless concentrations of $\mathrm{HBrO}_{2}$ and the metal ion catalyst, respectively. The Laplacian operator $\nabla^{2}=\partial^{2} / \partial x^{2}+\partial^{2} / \partial y^{2}$ for two spatial dimensions was used to simulate the thin film of solution. The parameters $q=10^{-4}$ and $\epsilon$, which was varied, were calculated from the concentrations in Table 1 and the FieldFörsterling ${ }^{31}$ rate constants according to the scaling of Tyson and Fife. ${ }^{5}$ Following Keener and Tyson, ${ }^{8}$ the stoichiometric factor $f=3$ was used to generate a nonoscillatory excitable system. Integrations were carried out by implicit solution of the kinetic term for $u$ and explicit solution of the kinetic term for $v$, following similar treatments of reaction-diffusion systems with stiff kinetics. ${ }^{32-34}$ The diffusion terms were integrated explicitly using a five-point formula, with the grid reflected at the edges and corners for no-flux boundary conditions. The spatial coordinates were scaled so that both diffusion coefficients $\left(D_{u}=D_{v}=2 \times\right.$ $10^{-5} \mathrm{~cm}^{2} \mathrm{~s}^{-1}$ ) were equal to unity (in dimensionless units).

A narrow channel of three grid points was defined through the middle of a wide barrier $(51 \times 201$ grid points) dividing the square area of $201 \times 201$ grid points into two equal parts. The width of the channel was set to a value slightly larger than the critical nucleation diameter, which was determined from eq 2 using the calculated planar wave velocity and a diffusion coefficient of $2 \times 10^{-5} \mathrm{~cm}^{2} \mathrm{~s}^{-1}$. Planar waves were initiated in one compartment at a period of $T_{\mathrm{f}}$ by setting the value of $u$ to 0.999 in a $3 \times 201$ strip of grid points for one time step.

Figure 9 shows the calculated excitation cycles of both the entering and exiting waves in a 1:1 pattern. The evolution of $u$ and $v$ at a grid point near the channel entrance was monitored, with the resulting cycle that of the planar wave. The effects of curvature are exhibited by the exiting wave, which was monitored one grid point beyond the exit. Because the dispersion of $u$ is enhanced in the curved front, its autocatalytic growth is retarded and the exiting wave is delayed at the end of the channel. The delay is seen in the excitation cycle as a distortion, where the trajectory approaches the unstable (middle) branch of the $u$ 


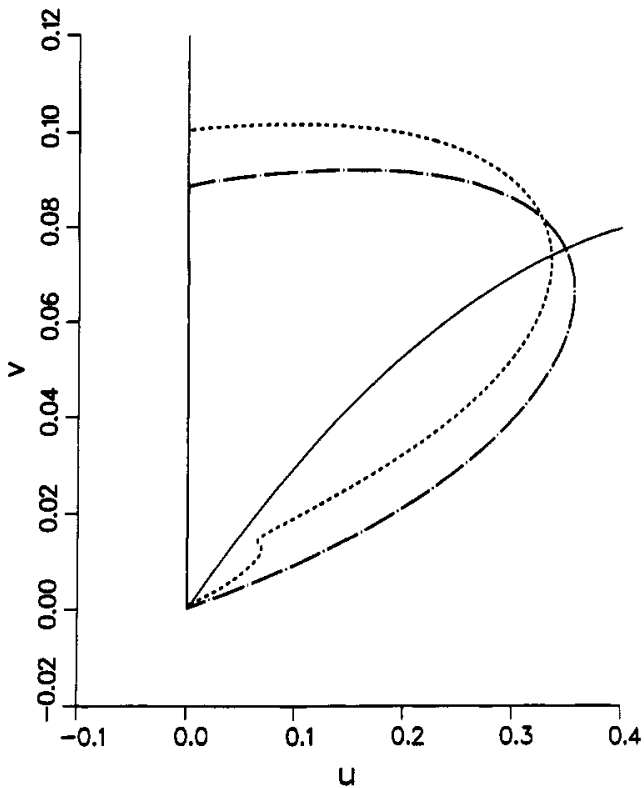

Figure 9. Calculated excitation cycle of entering $(-,-)$ and exiting (.-) waves for 1:1 pattern. The solid line shows the stable left branch and the unstable middle branch of $u$ nullcline (stable right branch not shown). Data were collected in line with the tube (positioned between grid points 76 and 126) at grid point 66 for entering waves and at grid point 127 for exiting waves. Parameters of eq 8: $\epsilon=0.0650, q=1 \times$ $10^{-4}$, and $f=3$. Dimensionless space unit $h=\left(10^{3}\right)^{1 / 2} \mathrm{~cm}^{-1} \times l$, dimensionless time unit $t=0.02 \mathrm{~s}^{-1} \times t^{\prime}$, where $l$ and $t^{\prime}$, respectively, are values of the space and time variables in physical dimensions. Edge length of square area of $201 \times 201$ grid points, $L=50.6$; step-size, $\Delta t$ $=5 \times 10^{-3}$; entering wave period, $T_{\mathrm{f}}=6.620$.


Figure 10. Concentration profile of $v$ as a function of time of entering (top) and exiting (bottom) waves for 1:2 pattern calculated from eq 8. Parameters and sampling points are the same as in Figure 9. Entering wave period $T_{f}=6.105$.

nullcline. Motion near this branch is slow, and the basic refractory period at the exit of the channel is longer than that for an element at the channel entrance. Even though the propagation of the wave is significantly delayed, the value of $T_{\mathfrak{f}}$ is sufficiently large that every entering wave brings about an above-threshold perturbation for wave initiation at the channel exit.

Figure 10 shows concentration profiles of $v$ at the entrance and exit of the channel with a slightly shorter period for the entering waves. Now, although every wave significantly perturbs the solution element at the channel exit, every other wave is ineffective in initiating a new wave. The result is a 1:2 resonance pattern.

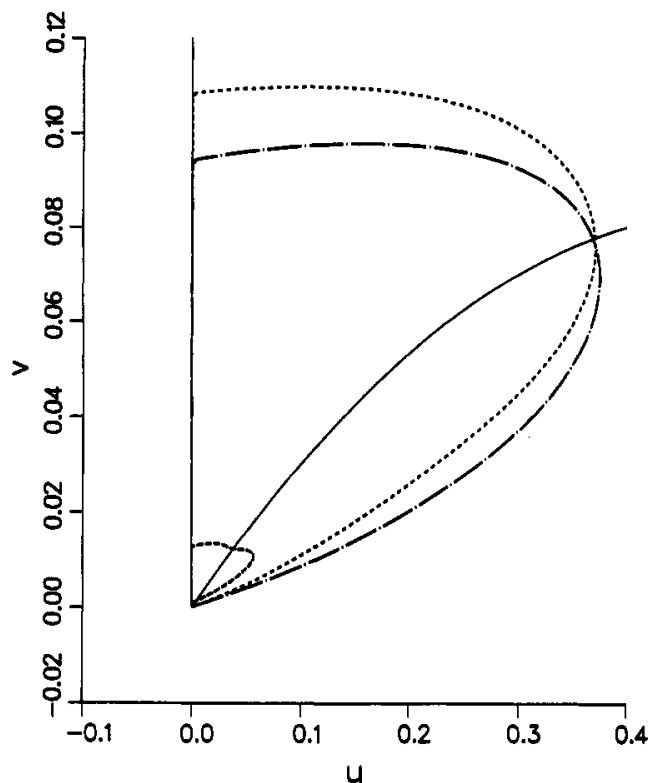

Figure 11. Excitation cycle of entering (- . - ) and exiting ( - . ) waves for 1:2 pattern. Calculation is the same as in Figure 10.

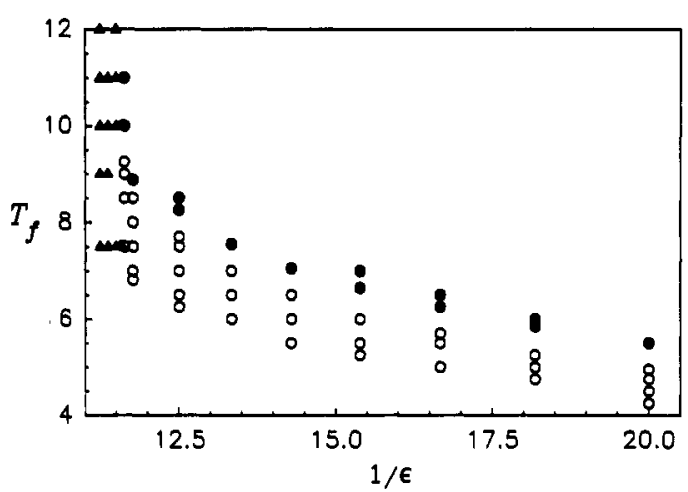

Figure 12. Phase diagram showing regions of resonance patterns for different values of period $T_{f}$ and $1 / \epsilon$. Resonance patterns are indicated by (waves exiting:waves entering) $0: 1(\Delta), 1: 1(0)$, and $1: 2(0)$. Calculation is the same as in Figure 9.

The corresponding excitation cycle in Figure 11 shows how the trajectory for the ineffective initiation follows a small excursion and quickly returns to the steady state. Of course, different trajectories are exhibited at different distances from the channel exit; at sufficiently large distances the ineffective perturbation will not be seen in the excitation cycle. It is also important to note that the perturbation threshold is not the middle branch of the $u$ nullcline as in the homogeneous system; rather, the effect of diffusive dispersion pulls the trajectory back from beyond the homogeneous threshold. The threshold also depends on the timing of the entering waves, increasing as $T_{\mathrm{f}}$ approaches $T_{\mathrm{B}}$. This has been referred to as a dynamic threshold by Sevčiková and Marek. ${ }^{35}$

The bromate concentration dependence in eq 8 occurs in the parameter $\epsilon$, which is inversely proportional to $\left[\mathrm{BrO}_{3}^{-}\right]$. A diagram similar to that in Figure 7 can therefore be constructed by varying the period $T_{f}$ of the entering waves for different values of $1 / \epsilon$. Figure 12 shows that as the value of $1 / \epsilon$ increases, the range in $T_{\mathrm{f}}$ for the 1:2 resonance pattern narrows, much like the trend in Figure 7 with increasing bromate concentration. It was not possible to generate entering waves with a sufficiently low period for the appearance of the $1: 3$ pattern using these parameter values. In addition, a much wider gap between the $1: 1$ and $1: 2$ patterns is exhibited, and new patterns appear in this gap. Figure 13 shows a bifurcation diagram for $1 / \epsilon=15.4$, where the firing number $f n$ is plotted as a function of $T_{\mathrm{f}}$. The concatenations of the $1: 1$ and $1: 2$ patterns can be arranged as one branch of a Farey tree, generated by adding the parent patterns according to Farey 


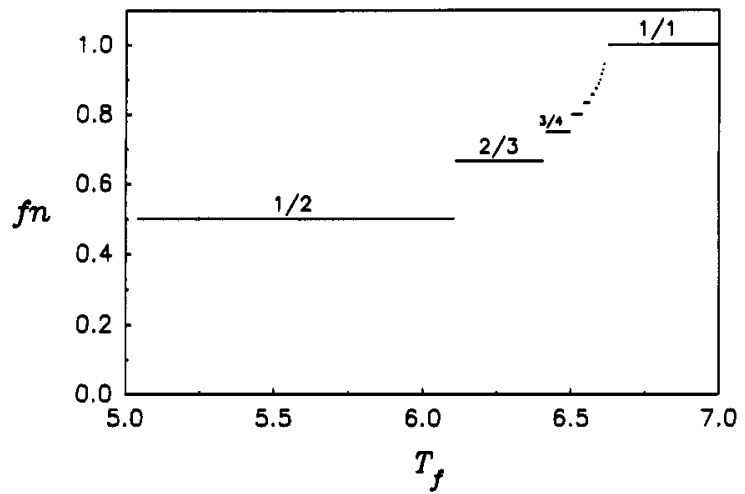

Figure 13. Bifurcation diagram showing firing number $\mathrm{fn}$ as a function of the entering wave period $T_{f}$, calculated as in Figure 12 with $1 / \epsilon=$ $1 / 0.0650 \approx 15.4$.

arithmetic. The occasional appearance of 2:3 patterns in the experiments with large tubes may be due to such mixing of $1: 1$ and $1: 2$ patterns.

Complex Resonances. The mapping procedure to describe the simple $1 / n$ resonances using eq 7 must be modified to describe the $(n-1) / n$ patterns shown in Figure 13 . For the $1 / n$ patterns, the basic refractory period $T_{\mathrm{B}}$ was defined by the largest value of $T_{f}$ for the 1:2 pattern, which is essentially the same as the value of $T_{\mathrm{f}}$ for the disappearance of the 1:1 pattern. The basic refractory period defined the "natural" length of the excitation cycle. In applying eq 7 , the phase is reset to zero on reaching the $\eta$ neighborhood of the steady state, where the system remains at rest until the next stimulus occurs. These rules are adequate for the $1 / n$ patterns, where the gap between each type of pattern is negligible; however, they are not appropriate for the patterns in Figure 13. The mapping procedure can be modified to describe both the $1 / n$ and $(n-1) / n$ patterns by taking into account an additional feature of the excitation cycle. It is apparent that the cycle is not complete when the $\eta$ neighborhood of the steady state is reached at time $T_{\mathrm{B}}$. In fact, the system continues to move along the slow manifold toward the steady state. In this region of phase space, the system is excitable and an entering wave always results in an above-threshold perturbation for wave initiation.

To account for the time spent by the system between $T_{\mathrm{B}}$ and the completion of the cycle, we define the $\theta \ll \eta$ neighborhood of the steady state. We denote $T_{\theta}$, the time required to return to the $\theta$ neighborhood, as the length of a "completed" excitation cycle. The dimensionless period of the entering waves is now defined by $\tau=T_{f} / T_{\theta}$, and the lower limit of the excitable zone is defined by $\varphi_{\mathrm{B}}=T_{\mathrm{B}} / T_{\theta}$, where $T_{\mathrm{B}}$ is the original basic refractory period. If $\varphi_{n} \leq \varphi_{\mathrm{B}}$, wave initiation due to an exiting wave does not take place. The system continues to move toward the steady state along the excitation cycle, and consequently $\varphi_{n+1}>\varphi_{n}$. However, if $\varphi_{\mathrm{B}}<\varphi_{n}<1$, the system is in the excitable zone of the excitation cycle. Now, wave initiation occurs before the cycle is completed, and $\varphi_{n+1}$ is therefore always less than $\varphi_{n}$ by exactly $(1-\tau)$.

On the basis of these definitions of the excitation cycle with an excitable zone, eq 7 can be modified to include the new feature:

$\varphi_{n+1}=\Psi\left(\varphi_{n}\right)= \begin{cases}g\left(\varphi_{n}\right)+\tau & \text { for } 0 \leq \varphi \leq \varphi_{\mathrm{B}} \\ g\left(\varphi_{n}\right)-(1-\tau) & \text { for } \varphi_{\mathrm{B}}<\varphi<1 \\ \tau & \text { for } \varphi \geq 1\end{cases}$

The third expression of eq 9 adds a constant in the mapping, which represents a phase-resetting event whenever $\varphi_{n+1} \geq 1$.

Figure 14 shows how successive iterations of eq 9 give rise to a 1:2 resonance pattern when $\tau<\varphi_{\mathrm{B}}$. We again assume a linear PEC to reflect the negligible phase shift caused by each exiting

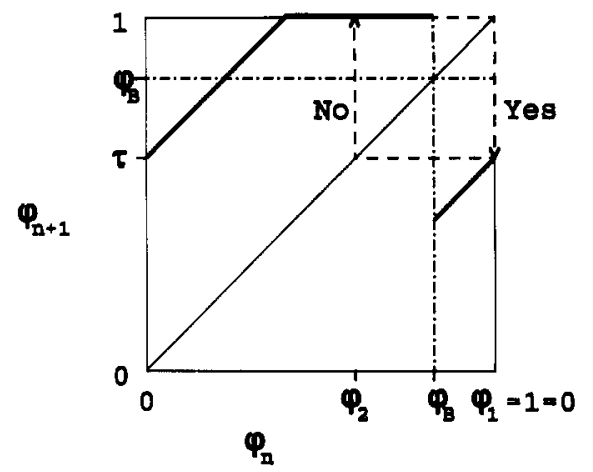

Figure 14. Successive iterations of map based on eq 9 for $1: 2$ resonance pattern.

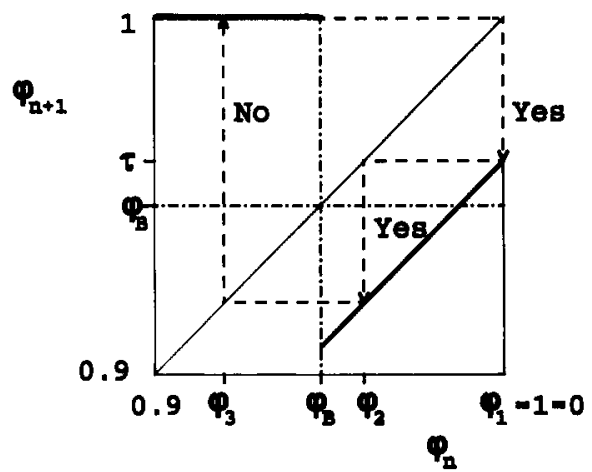

Figure 15. Successive iterations of map based on eq 9 for $2: 3$ resonance pattern.

wave. The particular resonance pattern is determined simply by the timing of the incoming waves relative to the position of the system on its excitation cycle. The evolution of the 1:2 pattern is described by the following iterations of the map:

$$
\begin{aligned}
\varphi_{1}=0 \underset{\text { Yes }}{\longrightarrow} \varphi_{2}=\tau \underset{\text { No }}{\longrightarrow} \varphi_{3}=1 \Rightarrow \varphi_{1} & =0 \underset{\text { Yes }}{\longrightarrow} \\
\varphi_{2} & =\tau \underset{\text { No }}{\longrightarrow} \text { etc... }
\end{aligned}
$$

Each vertical arrow corresponds to an incoming wave, with the upward arrow indicating an ineffective wave and the downward arrow indicating wave initiation following phase resetting. Thus the closed loop of dashed lines represents the periodic solution of the $1: 2$ resonance pattern.

When $\tau>\varphi_{B}$, the mapping always results in more complex resonance patterns. Figure 15 shows, for example, how successive iterations of the map give rise to a $2: 3$ pattern. In this case, there are three vertical arrows in the closed loop; two arrows point downward and one upward to give the 2:3 periodic solution. The resonance pattern is described by the following sequence of iterations:

$$
\begin{aligned}
\varphi_{1}=0 \longrightarrow \underset{\mathrm{Yes}}{\longrightarrow} \varphi_{2}=\tau \underset{\mathrm{Yes}}{\longrightarrow} \varphi_{3}=1-2(1-\tau) \underset{\text { No }}{\longrightarrow} \\
\varphi_{4}=1 \Rightarrow \varphi_{1}=0 \underset{\mathrm{Yes}}{\longrightarrow} \varphi_{2}=\tau \underset{\text { Yes }}{\longrightarrow} \text { etc... }
\end{aligned}
$$

As $\tau$ is increased further, more and more iterations must occur to leave the excitable zone, and a series of $(n-1) / n$ resonances develops between the 1:1 and 1:2 parent patterns.

Based on simple geometrical arguments, it is possible to define the period of the incoming waves $\tau=\tau_{n}$ at which a given $(n-$ $1) / n$ resonance pattern first appears (on decreasing $\tau$ ). We again assume that the dominant feature is the timing of the incoming waves $\left(\varphi^{\prime}=g(\varphi)=\varphi\right.$. For $(n-1) / n$ patterns with $n>2$, the 
TABLE 2: Period of Waves Entering Capillary Tube $\tau=\tau$, at Which a Particular $(n-1) / n$ Resonance Pattern First Appears on Decreasing $\tau$ (cf. Figure 13)

\begin{tabular}{cccc}
\hline resonance pattern & $T_{\mathrm{f}}$ & $\tau_{n}{ }^{\mathrm{a}}$ & $\tau_{n}{ }^{\mathrm{b}}$ \\
\hline $7 / 8$ & 6.585 & 0.995 & 0.989 \\
$6 / 7$ & 6.575 & 0.993 & 0.987 \\
$5 / 6$ & 6.560 & 0.991 & 0.985 \\
$4 / 5$ & 6.535 & 0.987 & 0.981 \\
$3 / 4$ & 6.495 & 0.981 & 0.974 \\
$2 / 3$ & 6.405 & 0.968 & 0.961
\end{tabular}

a Values of $\tau_{n}{ }^{2}=T_{t} / T_{b}$, where $T_{\theta}=6.62$, were obtained from numerical integration of eq 8; values of $\tau_{n}^{b}$ were calculated from eq 10 with $\varphi_{B}=$ 0.9222 .

bifurcation points are given by solving the following equation for $\tau_{n}$ :

$$
\left(\tau_{n}-\varphi_{\mathrm{B}}\right)=(n-2)\left(1-\tau_{n}\right)
$$

By choosing the lowest value of $T_{\mathrm{f}}$ for the 1:1 pattern in Figure 13 as the value of $T_{0}$, the bifurcation points for the appearance of each pattern can be predicted. Listed in Table 2 are values of $\tau_{\mathrm{n}}$ obtained from eq 10 and from the numerical integration of eq 8 . The values agree to within $1 \%$.

\section{Conclusion}

Measurements of wave transmission as a function of bromate concentration provide a highly reproducible value of the critical nucleation radius for a particular solution composition. There is a discrepancy, however, between the diffusion coefficient calculated from such measurements using the eikonal equation with the planar velocity and the anticipated value between 1.0 $\times 10^{-5}$ and $2.0 \times 10^{-5} \mathrm{~cm}^{2} \mathrm{~s}^{-1}$. This discrepancy arises because the velocity of a wave within the capillary tube is significantly higher than that of a planar wave in the bulk solution. When the wave velocity within the tube is used with the eikonal equation, a value of $1.27 \times 10^{-5} \mathrm{~cm}^{2} \mathrm{~s}^{-1}$ is calculated for the diffusion coefficient, which is within the range expected for small molecules. Apparently the chemical environment within and at the opening of the capillary tube differs from the bulk solution, perhaps due to the adsorption of bromine species. In view of the different methods employed in measuring the critical nucleation size in excitable BZ reaction mixtures, there is reasonably good agreement between the experimental determinations. ${ }^{10-12}$

The transmission of propagating waves through narrow gaps gives rise to resonance patterns similar to those found in periodically forced homogeneous systems. Such patterns may be of importance in certain types of biological signal transmission, e.g., propagating waves of electrical activity in the heart muscle. It is easy to imagine barriers of unexcitable tissue with narrow gaps that allow the passage of wave activity. How the transmitted waves - a particular resonance pattern determined by the size of the gap, the period of the incoming waves, and the excitability characteristics of the medium-interact with the surrounding unaffected wave activity could be of importance in heart maladies.

It is likely that more complex patterns occur for some values of parameters in the model system and for particular reactant concentrations in the experimental system. For example, the Farey mixing of the $1 / 2$ and $2 / 3$ patterns in Figure 13 to give $3 / 5$, $5 / 8$, etc. might occur for parameter values that widen the gap between the parent patterns. Such higher resonances would be reminiscent of mixed-mode or bursting patterns observed in oscillatory chemical systems ${ }^{36,37}$ and in neuronal firing. ${ }^{38}$ We also note that a single channel near the critical size represents a chemical analogue of the on-off switch; i.e., a chemical device that provides binary-coded output. An extension of the singletube system to multitube arrays that give rise to logic gates is currently being pursued. Such arrays can also be used in pathfinding algorithms, as recently demonstrated by Sepulchre and Babloyantz. ${ }^{39}$

Acknowledgment. We thank the National Science Foundation (Grants CHE-9222616 and INT-8822786) and the Hungarian Academy of Sciences for financial support of this work. We also thank John J. Tyson for enlightening discussions and his suggestion that wave nucleation could be studied with waves propagating through tubes. We thank Michael F. Crowley for his suggestions on mapping procedures for describing complex resonance patterns. V.G. is grateful to Richard J. Field for an invitation to present preliminary results of this project at the 47 th ACS Northwest Regional Meeting, Missoula, June 1992. Acknowledgment is made to the donors of The Petroleum Research Fund, administered by the American Chemical Society, for partial support of this research.

\section{References and Notes}

(1) Winfree, A. T. The Geometry of Biological Time; Springer: New York, 1980.

(2) Murray, J. D. Mathematical Biology; Springer: New York, 1989.

(3) Glass, L.; Mackey, M. C. From Clocks to Chaos; Princeton: New Jersey, 1988.

(4) Winfree, A. T. Faraday Symp. Chem. Soc. 1974, 9, 38.

(5) Tyson, J. J.; Fife, P. C. J. Chem. Phys. 1980, 73, 2224.

(6) Showalter, K.; Noyes, R. M.; Turner, H. J. Am. Chem. Soc. 1979, 101,7463 .

(7) Tyson, J. J.; Keener, J. P. Physica D 1988, 32, 327

(8) Keener, J. P.; Tyson, J. J. Physica D 1986, 21,307

(9) Keener, J. P. SIAM J. Appl. Math. 1986, 46, 1039.

(10) Nagy-Ungvárai, Zs.; Ungvárai, J.; Müller, S. C.; Hess, B. J. Chem. Phys. 1992, 97, 1004.

(11) Foerster, P.; Müller, S. C.; Hess, B. Science 1988, 241, 685.

(12) Foerster, P.; Müller, S. C.; Hess, B. Proc. Natl. Acad. Sci. U.S.A. 1989, 86, 6831.

(13) Pertsov, A. M.; Ermakova, E. A.; Shnol, E. E. Physica D 1990, 44, 178.

(14) Babloyantz, A.; Sepulchre, J. A. Physica D 1991, 49, 52.

(15) Sepulchre, J. A.; Babloyantz, A. Phys. Rev. Lett. 1991, 66, 1314.

(16) Kogan, B. Y.; Walter, J. K.; Billett, B. S.; Stevenson, W. G. Physica $D$ 1992, 59,275 .

(17) Field, R. J.; Noyes, R. M. J. Am. Chem. Soc. 1974, 96, 2001.

(18) Showalter, K. J. Phys. Chem. 1981, 85, 440.

(19) Wood, P. M.; Ross, J. J. Chem. Phys. 1985, 82, 1924.

(20) Kuhnert, L.; Krug, H.-J. J. Phys. Chem. 1987, $91,730$. 707.

(21) Nagy-Ungvárai, Zs.; Tyson, J. J.; Hess, B. J. Phys. Chem. 1989, 93,

(22) Mori, E.; Schreiber, I.; Ross, J. J. Phys. Chem. 1991, 95, 9359

(23) Kuhnert, L.; Krug, H.-J.; Pohlmann, L. J. Phys. Chem. 1985, 89, 2022.

(24) Dolnik, M.; Finkeová, J.; Schreiber, I.; Marek, M. J. Phys. Chem. $1989,93,2764$.

(25) Finkeová, J.; Dolnik, M.; Hrudka, B.; Marek, M. J. Phys. Chem. $1990,94,4110$.

(26) Dolnik, M.; Marek, M. J. Phys. Chem. 1991, 95, 7267.

(27) Doinik, M.; Marek, M.; Epstein, I. R. J. Phys. Chem. 1992, 96, 3218.

(28) Bak, P.; Bohr, T.; Jensen, M. H. In Directions in Chaos; Bai-Lin, H., Ed.; World Scientific: New Jersey, 1988; p 16, Vol. 2.

(29) Glass, L.; Shrier, A.; Béliar, J. In Chaos; Holden, A. V., Ed.; Princeton: New Jersey, 1986; p 237.

(30) Field, R. J.; Noyes, R. M. J. Chem. Phys. 1974, 60, 1877.

(31) Field, R. J.; Forsterling, H.-D. J. Phys. Chem. 1986, 90, 5400.

(32) Barkley, D. Physica D 1991, 49,61.

(33) Vasquez, D. A. J. Comput. Chem. 1992, 13, 570.

(34) Gáspár, V.; Maselko, J.; Showalter, K. Chaos 1991, 1, 435

(35) Sevčiková, H.; Marek, M. Physica D 1991, 49, 114

(36) Sørensen, P. G. Faraday Symp. Chem. Soc. 1974, 9, 88

(37) Petrov, V.; Scott, S. K.; Showalter, K. J. Chem. Phys. 1992, 97,6191.

(38) Kandel, E. R.; Schwartz, J. H.; Jessell, T. M. Principles of Neural Science, 3rd ed.; Elsevier: New York, 1991.

(39) Sepulchre, J. A.; Babloyantz, A.; Steels, L. In Proc. Int. Conf. Art. Neur. Net.; Simula, O., Ed.; Elsevier: Amsterdam, 1991. 